\title{
Excitation spectroscopy of single quantum dots at tunable positive, neutral and negative charge states
}

\author{
Y. Benny ${ }^{*}$ Y. Kodriano, E. Poem, and D. Gershoni \\ The Physics Department and the Solid State Institute, \\ Technion - Israel Institute of Technology, Haifa 32000, Israel. \\ T. A. Truong and P. M. Petroff \\ Materials Department, University of California, Santa Barbara, California 93106, USA
}

(Dated: November 8, 2018)

\begin{abstract}
We present a comprehensive study of the optical transitions and selection rules of variably charged single self-assembled InAs/GaAs quantum dots. We apply high resolution polarization sensitive photoluminescence excitation spectroscopy to the same quantum dot for three different charge states: neutral and negatively or positively charged by one additional electron or hole. From the detailed analysis of the excitation spectra, a full understanding of the single-carrier energy levels and the interactions between carriers in these levels is extracted for the first time.
\end{abstract}

\section{INTRODUCTION}

The discrete nature of the energy levels in semiconductor quantum-dots (QDs) has been a subject of many studies 17. This "atomic-like" spectral feature of QDs, together with their compatibility with modern semiconductor-based microelectronics and optoelectronics, make QD-based devices particularly promising as building blocks for future technologies involving quantum information processing (QIP) ${ }^{8-10}$ Devices that emit single and entangled photons on demand 115 are typical examples of these potential applications. One important reason which makes QDs particularly attractive for these applications is that, unlike atoms, their charge state can be easily controlled by external fields. 16

A detailed understanding of the energy levels of confined carriers in these QDs, and the interactions between them, is essential for implementing these potential applications. Therefore, there are many experimental and theoretical studies aiming at achieving this goal.3|4|19|20| Various experimental methods are applied in these studies: polarization sensitive photoluminescence $(\mathrm{PL}) \sqrt[3|4| 6 \mid 21]{ }, \mathrm{PL}$ in the presence of electric ${ }^{3 / 4}$ and magnetic fields 22 , and PL excitation (PLE) $)^{5 / 7 / 23}$ spectroscopies, second order intensity correlation measurements $6[21]$ and time resolved spectroscopy 2124 , just to mention a few. These various experimental methods, when combined with many body models, lead to a relatively good understanding of the

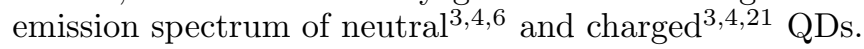
In a recent study, we applied one and two photon PLE spectroscopies to a neutral QD to fully reveal its excitonic and biexcitonic resonance-rich spectrum. ${ }^{7}$

In the current work, we use our ability to optically control the QD charge state in order to apply high spectral resolution polarization sensitive PLE spectroscopy of the same QD at different charge states. A detailed understanding of the spectrum of a neutral $\mathrm{QD}^{7}$ leads in turn to an understanding of the spectrum when the QD is charged with an additional, single electron or heavyhole. This way, a comprehensive understanding of sin- gle electron and single hole states and energy levels is achieved, together with characterizations of the interactions between carriers in these levels.

\section{THEORY AND EXPERIMENT}

\section{A. The experimental setup}

The sample used in this work was grown by molecularbeam epitaxy (MBE) on a (001) oriented GaAs substrate. One layer of strain-induced $\operatorname{In}_{\mathrm{x}} \mathrm{Ga}_{1-\mathrm{x}} \mathrm{As}$ QDs was deposited in the center of a one wavelength microcavity. The microcavity was designed to have a cavity mode which matches the QD emission due to ground state e-h pair recombinations. Such microcavity significantly improves photon collection efficiency from emission lines which resonate with the cavity mode since their light is emitted at normal incidence. The light from higher energy lines is emitted at energy dependent angle. Therefore, the spectral window in which light emitted from within the cavity can be efficiently collected is defined by the numerical aperture (NA) of the collecting optics. 25 Planar microcavity, however, affects only marginally the radiative rate of emission lines (a few percents Purcell effect) and therefore the intensity of various emission lines within the allowed spectral window is not influenced by the cavity. Absorption resonances measured by PLE spectroscopy are typically above this window, in a spectral range where the upper DBR mirror has high reflectivity (about 99 percent). Thus, laser light coupling is equally inefficient for all the absorption lines within the stop band of the DBR mirror.

During the growth of the QD layer, the sample was not rotated, resulting in a gradient in the grown QD density. The estimated QD density in the sample areas that were measured is $10^{8} \mathrm{~cm}^{-2}$. However, the density of QDs that emit in resonance with the microcavity mode is more than two orders of magnitude lower.26 Thus, single QDs separated by a few tens of micrometers were 
easily located by scanning the sample surface during PL measurements. Strong anti-bunching in intensity autocorrelation measurements was then used to verify that the isolated QDs are single dots and that they are single photon sources.

The sample was placed inside a sealed metal tube immersed in liquid helium, maintaining a temperature of $4.2 \mathrm{~K}$. A $\times 60$ microscope objective with a numerical aperture of 0.85 was placed above the sample and used to focus the excitation laser on the sample surface and to collect the emitted PL. The relatively high NA of our system resulted in $\sim 15 \mathrm{meV}$ broad spectral window above the cavity mode energy in which PL emission from the QDs was efficiently collected. We used a $\mathrm{cw}$ tunable Ti:sapphire laser to scan the energy of the optical excitation. The laser emission energy could be continuously changed using coordinated rotations of a three plate birefringent filter and a thin etalon. The excitation intensity was about $0.5 \mu$ Watt, in which no power broadening of the absorption resonances, was observed, but the PL emission was reasonably intense, allowing efficient PLE spectroscopy. The polarization of the light was adjusted and analyzed using a polarized beam splitter (PBS) and two pairs of computer-controlled liquid crystal variable retarders (LCVRs). The PL was spectrally resolved by a 1-meter monochromator and detected by a cooled CCD camera. ${ }^{7}$

For the polarization sensitive PLE spectroscopy, we monitored the polarized emission from an identified PL spectral line while varying the energy and polarization of the exciting light source. From the measured variations in the intensity of the emitted PL we construct the PLE spectrum. Resonances in the PLE spectrum are due to many carrier states in which a photon is absorbed by generating an additional electron-hole pair. Increased absorption results in increased emission intensity from the respective PL lines due to recombination of an electronhole pair from a many carrier state to which the excited carriers are relaxed prior to their recombination. The polarization sensitivity is due to one to one correspondence between the many carriers' spin wavefunction and the polarization of the absorbed or emitted photon. ${ }^{217}$ The energy of a particular resonance, its relative intensity, the particular lines that it relaxes to, and its polarization selection rules are then used to unambiguously identify the many-carrier states which form this resonance. ${ }^{727 / 28}$

Variation in the QD charge state was achieved by additional excitation with very weak intensity light with an energy above the bandgap. We discovered that the average charge state of the QD strongly depends on the energy of this minute amount of light. By changing the light color from red $(633 \mathrm{~nm})$ to violet $(458 \mathrm{~nm})$ we succeeded to vary the QD charge state from positive to negative, respectively. For each color, the average charge was different, as judged by the intensity of the emission from various charged exciton states. By mixing two colors, any desired average charge state can be obtained. While the exact mechanism of this charging control method is not accurately known, it can be qualitatively explained in terms of deionization of ionized impurity centers in the vicinity of the QD.29130 A more quantitative description of this controlled charging is currently under study, however, it is beyond the scope of this work.

It is important to note that the charge state of the QD achieved by this weak high energy illumination is an average state only. Typically, we observe emission from at least two, more often three different charge states at a given steady state illumination condition. Clearly, under these conditions the charge state of the QD fluctuates in time. The fluctuation times are excitation intensity dependent and can be straight forwardly measured using second order intensity correlation measurements. We recently reported on characteristic optical charging times of few nanosecond $\$ 31$ and on a mechanism of charge fluctuations via the dark exciton state. In the current work, the intensity of the high energy excitation was much lower, resulting in at least an order of magnitude longer charging times. Since the PL accumulation times were typically about a second, charge fluctuations times are still too short to cause observable "telegraphic" noise in the measurements.

\section{B. Photoluminescence spectra}

Fig. 1 presents rectilinear horizontal $(\mathrm{H})$ and vertical (V) polarized PL spectra of a single QD in resonance with the microcavity mode for three different average charge states. The observed spectral lines are identified in the Figure.

The notation used by Benny et al.$^{7}$ is used in this work as well: A single carrier state is described by its envelope wavefunction or orbital mode $(\mathrm{O}=1,2, \ldots, 6)$, where the number represents the energy order of the level, so that $\mathrm{O}=1$ represents the ground state. $\mathrm{O}$ is followed by the type of carrier, electron (e) or heavy-hole (h) and a superscript which describes the occupation of the single carrier state. The superscript can be either 1 (open shell) or 2 (closed shell), subject to the Pauli exclusion principle (unoccupied states are not included in the description). All the occupied states of carriers of same type are then marked by subscripts which describe the mutual spin configuration $(\sigma)$ of these states. A full description of a positive trion with two unpaired holes has therefore the form $\left(\mathrm{O}_{\mathrm{e}_{1}} \mathrm{e}^{1}\right)_{\sigma_{\mathrm{e}}}\left(\mathrm{O}_{\mathrm{h}_{1}} \mathrm{~h}^{1} \mathrm{O}_{\mathrm{h}_{2}} \mathrm{~h}^{1}\right)_{\sigma_{\mathrm{h}}}$ and in the same way a negative trion with two unpaired electrons has the form $\left(\mathrm{O}_{\mathrm{e}_{1}} \mathrm{e}^{1} \mathrm{O}_{\mathrm{e}_{2}} \mathrm{e}^{1}\right)_{\sigma_{\mathrm{e}}}\left(\mathrm{O}_{\mathrm{h}_{1}} \mathrm{~h}^{1}\right)_{\sigma_{\mathrm{h}}}$.

Fig. 1(a) presents the PL emission spectrum of the QD populated on average with one positive charge. This PL was obtained while the QD was excited by a $\mathrm{HeNe}$ laser light. The spectrum in this case is dominated by an unpolarized spectral line which appears $0.4 \mathrm{meV}$ below the neutral exciton line. Based on previous studies, we unambiguously identify this spectral line as the optical transition from the ground state positive trion $\left(X^{+1}\right)$, $\left(1 e^{1}\right)\left(1 h^{2}\right) \rightarrow\left(1 h^{1}\right)$ to a single ground level heavy hole 
state ${ }^{21}$ In the initial state, the two holes form a singlet of spin 0 in their ground level. The total spin of this state is therefore given by the spin-half state of the singleelectron. As expected, this total half-integer spin state is doubly (Kramers) degenerate in the absence of external time reversal breaking perturbation, such as a magnetic field.

The selection rules for optical transitions from this state are determined by the spin of the recombining electron ${ }^{[32}$ A spin-up, $\uparrow($-down, $\downarrow)$ electron recombines with a spin-down, $\Downarrow$ (-up, $\Uparrow)$ hole to give a left (right) hand circularly polarized photon with angular momentum of -1 $(+1)$. Since excitation at this high, above bandgap energy, photogenerates an equal mixture of the two electron spin states, the PL that results from the recombination is unpolarized.

The energy separation between the first and second hole levels is smaller than that between the corresponding electron levels, mainly due to the hole's larger effective mass. The lowest energy excited level of the positive trion is therefore one in which one of the holes is in its first excited level. 21 The total spin of the two holes that occupy the QD is 3, and thus there are four possible states with three possible spin projections for the two hole total spin; $+3,(-3)$ when both holes are aligned with spin up (down), and two states with total spin projection 0 , when the holes spins are anti-aligned. The hole-hole exchange interaction removes the degeneracy between the singlet state, $1 / \sqrt{2}(\Downarrow \Uparrow-\Uparrow \Downarrow)\left(S^{h}\right)$, which is antisymmetric under carrier exchange, and the three, lower energy, sym-

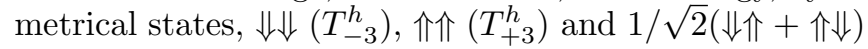
$\left(T_{0}^{h}\right)$. There is experimental evidence, based on recombination of the doubly positively charged exciton, that the degeneracy of the triplet states is further removed to a higher energy singlet with spin projection 0 and a lower energy doublet with spin projection $\pm 3{ }^{\frac{4}{4}}$ Here, in the case of the excited positive trion, this degeneracy is, in any case, removed by the electron-hole exchange interaction with the single electron.

The effect of the electron-hole exchange interactions on the triplet states of a trion are well described elsewhere in terms of the pseudospin Hamiltonian ${ }^{2133}$ Fig. 2 describes the energies of these eigenstates. The emission and absorption transitions of the positively charged trion are described in the Figure as well. For simplicity, these transitions are denoted by numbered arrows where the numbers reflect their energy order and up (down) arrow symbolizes photon absorption (emission). In a simple model, optical transitions between electron and hole levels of different orbital symmetries are forbidden, due to zero overlap between the electron and hole envelope wavefunctions. ${ }^{[3}$ However, our experimental results clearly indicate that these transitions are allowed, probably due to symmetry breaking between the electron and hole potentials. $\frac{523}{5 .}$

We note in Fig. 2 that the optically allowed transitions from the triplet states are partially rectilinearly polarized, due to the mixing between the $T_{ \pm 3}^{h}$ and $T_{0}^{h}$ states, described by the coefficients $\alpha$ and $\beta{ }^{[2] 33}$ These two transitions are identified in the PL spectrum, where the transition from the $T_{ \pm 3}^{h}$ state appears at $-4.06 \mathrm{meV}$ and the transition from the $T_{0}^{h}$ state appears at $-4.17 \mathrm{meV}{ }^{21} \mathrm{At}$ an energy of $0.43 \mathrm{meV}$, about $4.6 \mathrm{meV}$ higher, the transition from the singlet $\left(1 e^{1}\right)\left(1 h^{1} 2 h^{1}\right)_{S} \rightarrow\left(2 h^{1}\right)$ is identified. The transitions from the positive ground state biexciton, $\left(1 e^{2}\right)\left(1 h^{2} 2 h^{1}\right)$, to these triplet states are identified in the spectrum as well. These biexcitonic transitions were identified previously by time-resolved intensity correlation measurements. ${ }^{21}$ Additional lines are identified as transitions related to a two-hole charged QD $\left(X^{+2}\right)$. These transitions present exactly the same resonances in their PLE spectra (not shown here), and thus we attribute them to emission lines that occur from the same initial state.

Fig. 1(b) presents the PL spectrum excited with 488 $\mathrm{nm} \mathrm{Ar}+$ laser light. Under these conditions the QD is on average neutral. One clearly notes that neutral optical transitions in this spectrum are stronger than transitions in the presence of additional charge. The ground state neutral exciton and biexciton are readily identified by their well studied fine-structure cross-rectilinearly polarized doublets $\sqrt[3435]{35}$ The biexciton doublet, about $3.3 \mathrm{meV}$ lower in energy than the exciton doublet, has the same energy separation between its two cross-rectilinearly polarized components as the exciton albeit with reversed order, as expected ${ }^{3 / 7}$ Additional neutral transitions are observed, resulting from the spin-blockaded hole triplet states of the first excited biexciton, where one hole is in the second orbital energy mode. These lines are discussed in detail elsewhere ${ }^{6 / 7}$ The transitions from the neutral triexciton, where the $\mathrm{QD}$ is populated by three electrons and three holes, $\left(1 e^{2} 2 e^{1}\right)\left(1 h^{2} 2 h^{1}\right)$, to the neutral electron-triplet-hole-triplet biexciton states ${ }^{7}$ are identified in this spectrum as well.

Fig. 1(c) presents the PL spectrum excited by $458 \mathrm{~nm}$ $\mathrm{Ar}+$ laser light. Under these excitation conditions, the QD is on average negatively charged with one electron. The optical transitions in which the QD is negatively charged become much stronger than the neutral transitions, while positive transitions nearly vanish. The eigenstates of the negatively charged QD can be described in a similar way to those of the positively charged one. Here, the PL spectrum is dominated by an unpolarized spectral line due to recombination from the ground state of the negative trion, $\left(1 e^{2}\right)\left(1 h^{1}\right) \rightarrow\left(1 e^{1}\right)$. In addition, the transitions from the metastable, spin-blockaded excited trion levels, where one electron occupies the first excited electron orbital level, are observed. Similarly to the positive trion, four different spin configurations are expected; one anti-symmetric with respect to electron exchange with total spin projection $0,1 / \sqrt{2}(\uparrow \downarrow-\downarrow \uparrow)\left(\mathrm{S}^{\mathrm{e}}\right)$, and three symmetric triplet states with total spin projection $0,1 / \sqrt{2}(\uparrow \downarrow+\downarrow \uparrow)\left(\mathrm{T}_{0}^{\mathrm{e}}\right)$, and $1(-1): \uparrow \uparrow\left(T_{+1}^{e}\right)[\downarrow \downarrow$ $\left.\left(T_{-1}^{e}\right)\right]$. Due to the electron-electron exchange interaction the singlet state is higher in energy by a few $\mathrm{meV}$, and the electron-hole exchange interaction further removes 


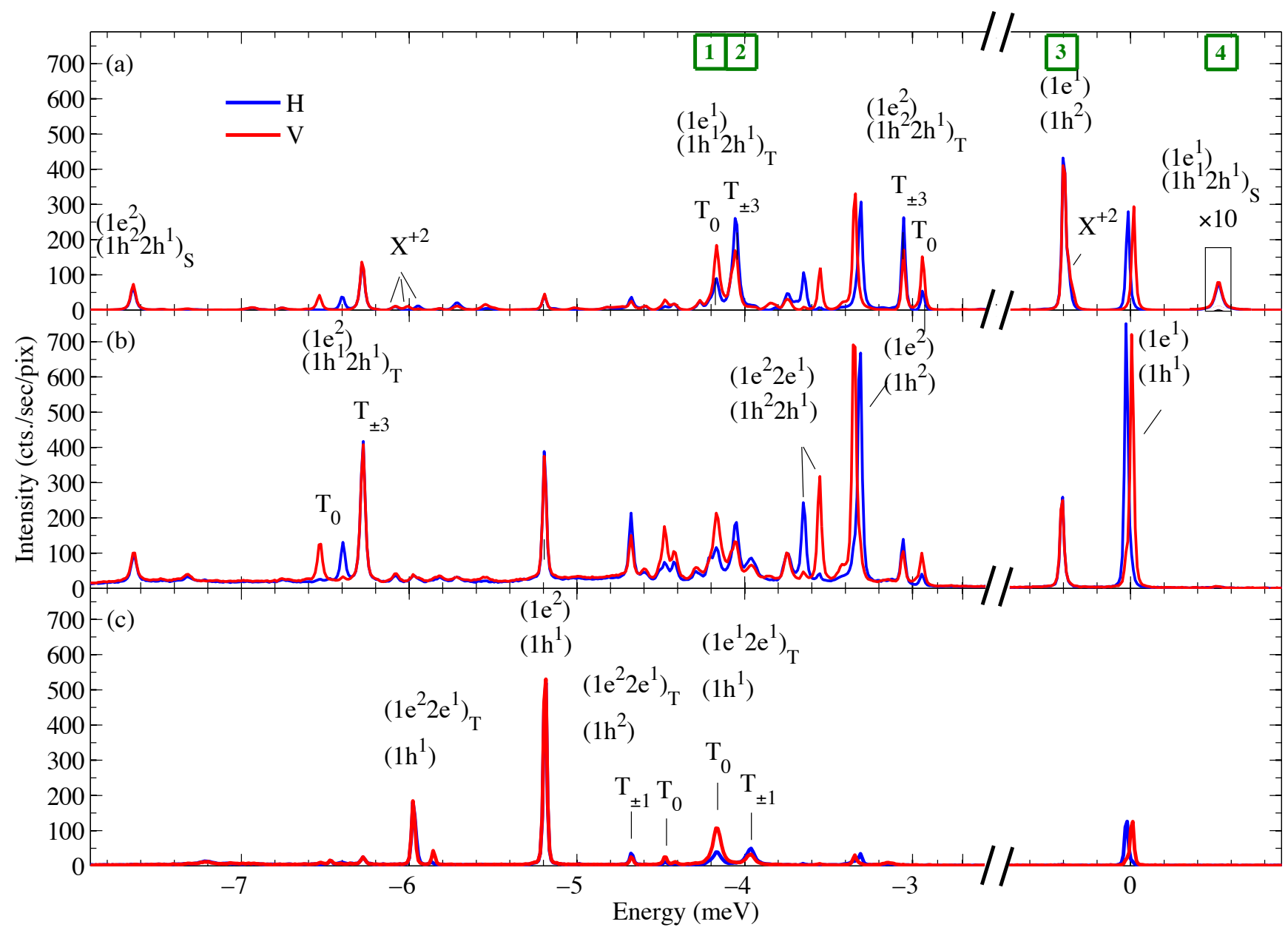

FIG. 1: Rectilinearly polarized horizontal (blue line) and vertical (red line) PL spectra of the single QD, at various charge states. In (a) the QD is on average positively charged by an additional heavy hole, in (b) it is neutral, and in (c) it is charged negatively, having on average one additional electron. The spectral lines due to excitonic and biexcitonic recombinations are identified in the Figure, denoted by the initial state of the optical transition. The numbers in bold rectangles in panel (a) denote optical transitions with the same numbers as in Fig. 2

the degeneracy between the triplet states.

The transitions from the ground state of the negatively charged biexciton to the metastable spin-blockaded triplet states of the exciton are also observed in the PL spectrum of Fig. 11(c). The identification of these biexcitonic transitions was confirmed by time-resolved intensity correlation measurements between the cascading transitions in a similar manner to the positive transitions 21 (not shown here). The intense line at about $-6 \mathrm{meV}$ is observed only in the PL spectrum of the negatively charged QD state. We attribute this line to a transition from a doubly negatively charged QD $\left(1 e^{2} 2 e^{1}\right)\left(1 h^{1}\right) \rightarrow$ $\left(1 e^{1} 2 e^{1}\right)_{T}\left(X^{-2}\right)$.

\section{Photoluminescence excitation spectra}

Higher energy transitions were studied by PLE measurements. The main resonances in the PLE can be easily understood by inspecting the simple state diagrams presented in Fig. 3 . In these diagrams the initial carriers are presented in grey and the resonantly photogenerated electron-hole pair is presented in red. Each row in Fig. 3 corresponds to a certain photoexcited pair, and each column corresponds to a particular QD charge occupation.

The first row of Fig. 3(a) describes various transitions in which a ground-state electron and a first-excited-state hole, $\left(1 e^{1}\right)\left(2 h^{1}\right)$, are photogenerated. If the pair is added to an empty $\mathrm{QD}$, an exciton will be formed $\left(X^{0}\right)$. If the QD is populated by a single electron, the added pair will result in a negative trion $\left(X^{-1}\right)$ in which the two electrons are paired in the ground state (singlet). If the $\mathrm{QD}$ is populated by a hole, four different Kramers degenerate spin-configurations of a positively charged trion $\left(X^{+1}\right)$ may be formed: three metastable spin-blockaded states in which the holes form triplets, and one state in which the holes form a singlet state. If the QD is occupied by an exciton, then the added pair will result in one of the 


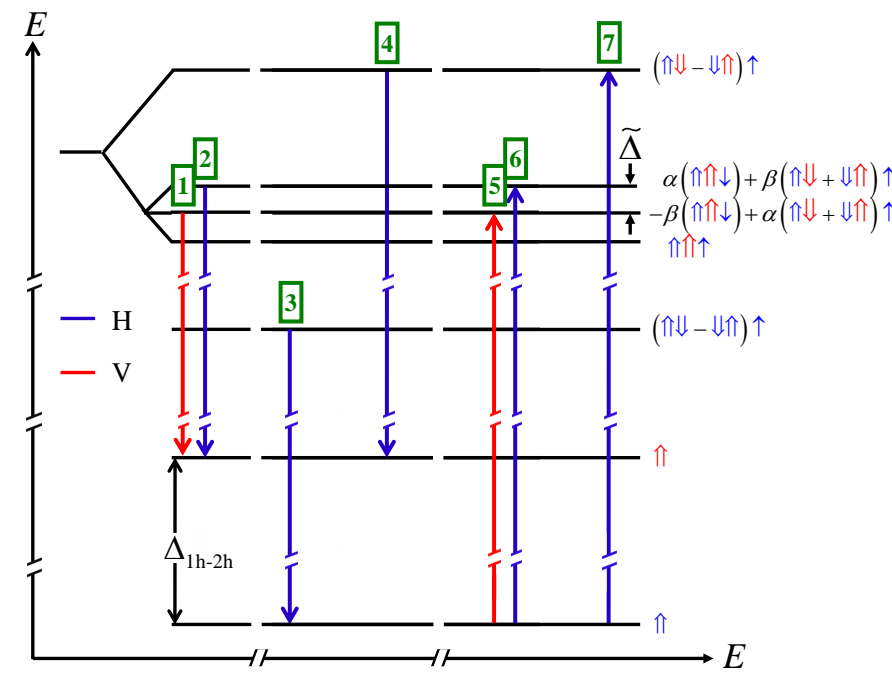

FIG. 2: Energy diagram of the positively charged trion states, $\left(1 e^{1}\right)\left(1 h^{2}\right)$ and $\left(1 e^{1}\right)\left(1 h^{1} 2 h^{1}\right)$ and the optical transitions to and from these states. Blue (red) arrows represent partial $\mathrm{H}(\mathrm{V})$ linear polarization. ${ }^{2}$ The optical transitions are numbered in increasing energy order, and the numbers corresponds to transitions observed in PL (Fig.1) and PLE (Fig.4) spectra. The spin configurations are presented to the right. Blue single (double) arrows represent a ground state electron (hole) and red single (double) arrow is for an excited electron (hole). $\alpha$ and $\beta$ are the coefficients of mixing between the $T_{ \pm 3}$ and $T_{0}$ states.

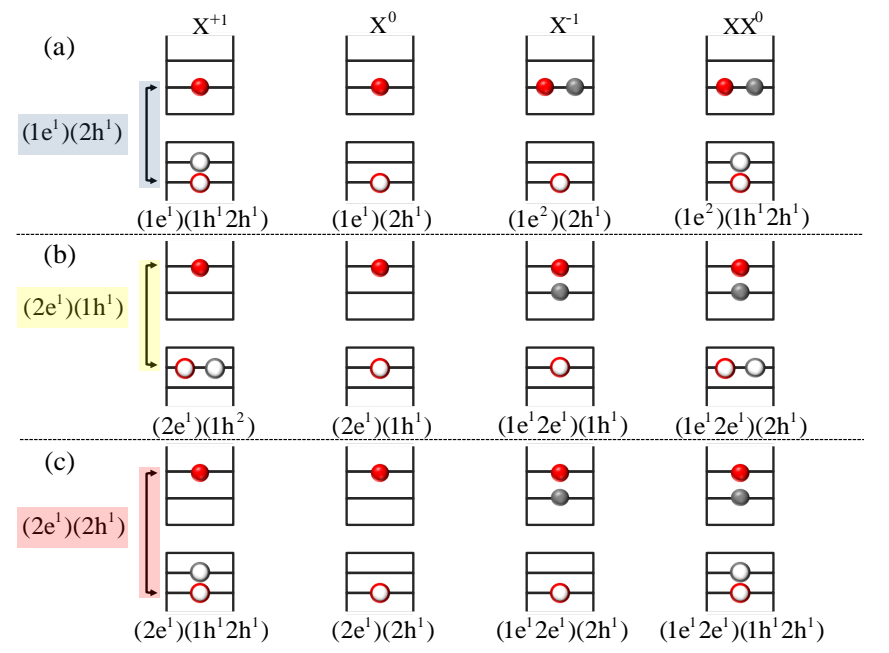

FIG. 3: Schematic descriptions of the multi-carrier resonances observed in the PLE spectra (Fig. 4). Optical transitions in which $1 e^{1}-2 h^{1}, 2 e^{1}-1 h^{1}$ and $2 e^{1}-2 h^{1}$ electronhole pair are photogenerated are described in (a), (b) and (c), respectively. Transitions involving the positive trion, the neutral exciton, the negative trion and the neutral biexciton are presented, in the first, second, third and fourth column, respectively. The background colors are used for identifying these transitions in Fig. 4. Filled (empty) red circle represents the photogenerated electron (hole) and a filled (empty) gray circle represents a resident electron (hole). neutral biexcitonic $\left(X X^{0}\right)$ e-singlet-h-triplet states. The transitions described in this row are marked blue.

In the second row, Fig. 3(b), the photogenrated electron is in its second energy level and the photogenerated hole is in its ground state $\left(2 e^{1}\right)\left(1 h^{1}\right)$. In general, these resonances are expected at higher energies than the corresponding resonances (same column) in Fig. 3(a), because the separation between the energy levels of the electrons is larger than that of the heavy holes. There is, however, one important difference between the two cases. The energy difference between the first two electron levels closely resonates with the energy of one longitudinal optical ( $\mathrm{LO}$ ) phonon in the materials composing the QD and the wetting layer. This results in efficient LO phonon mediated coupling between these electronic levels. This coupling has important consequences on the observed spectra, as previously noted ${ }^{7 / 36}$ and further discussed below. The transitions described in this row are marked yellow.

In the third row, Fig. 3(c), both photogenerated carriers, the electron and hole, are in their first excited state, $\left(2 e^{1}\right)\left(2 h^{1}\right)$. Thus, these transitions are at higher energies than the transitions in rows (a) and (b). The trions in this case have four Kramers' degenerate spin configurations of the majority carriers, as in the case of rows (a)(b); three triplet states and one singlet state. One of the four levels, that in which all the carriers spins are aligned, is optically inaccessible. The neutral biexciton, however, has sixteen spin configurations $\left(2^{4}\right)$ : e-triplet-h-triplet (nine states), e-triplet-h-singlet (3 states), e-singlet-htriplet (3 states) and e-singlet-h-singlet (1 state). Here as well, two states in which all the carriers' spins are aligned are optically inaccessible. ${ }^{[7}$ The LO phonon mediated coupling between the first two electronic levels, has similar signature in the PLE spectra from Fig. 3(c) as it has in the PLE spectra of Fig. 3(b). The transitions described in this row are red.

In Fig. 4, we present the PLE spectra of the main optical transitions presented in Fig. 1, together with a high resolution polarization sensitive PL spectra of the spectral lines used for monitoring the PLE spectra.

Fig. 4(a) and Fig. 4(b) present the PLE spectra of the neutral exciton and biexciton, respectively. These spectra were presented and discussed previously! ${ }^{7}$ Here they are displayed for comparison with the PLE spectra of the charged trions. The PLE spectra of the ground and first excited positive trion are presented in Fig. 4(c) and Fig. 4(d), respectively. Similarly, the PLE from the ground and first excited negative trion are presented in Fig. 4(e) and Fig. 4(f), respectively. The identified resonances are denoted in each spectrum.

The transitions that we describe in Fig. 3 are clearly identified in the PLE spectra in Fig. 4(a-f), where they are colored according to their classification. We note that transitions in which the hole is excited to its $2 \mathrm{nd}$, 3rd and 4th energy levels are similar in nature. Therefore they are all marked blue in Fig. 4. The identifications of the various lines are based here on their energetic or- 


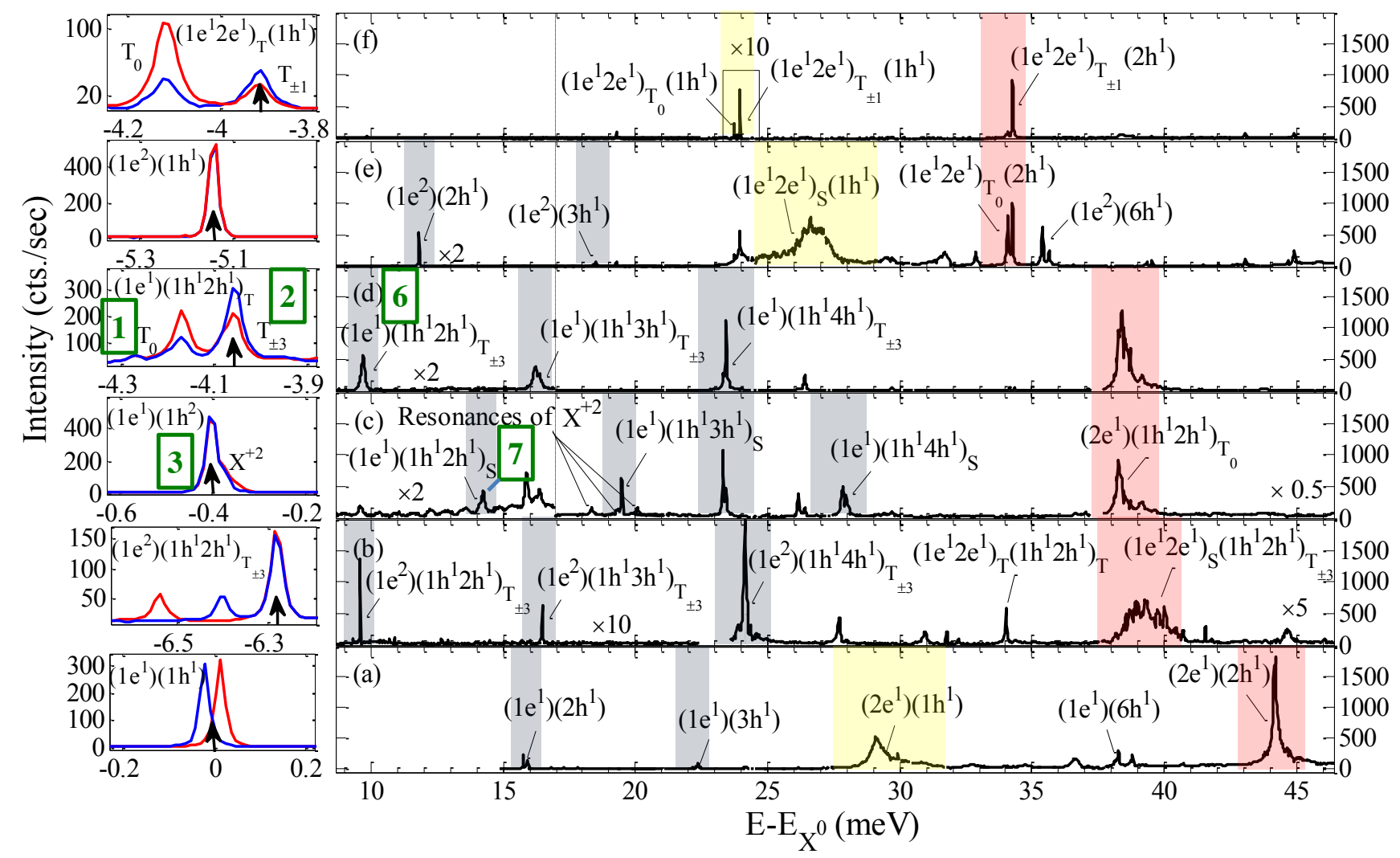

FIG. 4: Measured linear horizontal (blue) and vertical (red) polarized PL (left panels) and total linear polarized PLE (right panels) spectra of the transitions $\left(1 e^{1}\right)\left(1 h^{1}\right) \rightarrow 0(\mathrm{a}),\left(1 e^{1}\right)\left(1 h^{1} 2 h^{1}\right)_{T_{ \pm 3}} \rightarrow\left(1 e^{1}\right)\left(2 h^{1}\right)(\mathrm{b}),\left(1 e^{1}\right)\left(1 h^{2}\right) \rightarrow\left(1 h^{1}\right)(\mathrm{c})$ $\left(1 e^{1}\right)\left(1 h^{1} 2 h^{1}\right)_{T_{ \pm 3}} \rightarrow\left(2 h^{1}\right)(\mathrm{d}),\left(1 e^{2}\right)\left(1 h^{1}\right) \rightarrow\left(1 e^{1}\right)(\mathrm{e})$ and $\left(1 e^{1} 2 e^{1}\right)_{T_{ \pm 1}}\left(1 h^{1}\right) \rightarrow\left(2 e^{1}\right)(\mathrm{f})$. The assignment of the resonances are marked on the figures, above the observed resonances. The numbers in bold rectangles in panels (c) and (d) denote optical transitions with the same numbers in Fig. 2 .

der, their oscillator strength, and the emission line which monitored their absorption. In addition we utilized the similarities between the spectra from charged states to that from the neutral state ${ }^{7}$ of the same QD. We show below that polarization sensitive PLE measurements further support our line identifications.

By inspecting Fig. 2 one immediately sees that the energy difference between the first and second hole orbitals, $\Delta_{1 h-2 h}$, can be directly extracted from the energy difference between the absorption transitions 5,6 and 7 $\left[\left(1 h^{1}\right) \rightarrow\left(1 e^{1}\right)\left(1 h^{1} 2 h^{1}\right)_{\sigma_{h}}\right]$ and the emission transitions 1,2 and $4,\left[\left(1 e^{1}\right)\left(1 h^{1} 2 h^{1}\right)_{\sigma_{h}} \rightarrow\left(2 h^{1}\right)\right]$, respectively. Thus, using Figs. 4 (c,d), [transition 5 is seen in the PLE spectrum of the PL from the line $\left(1 e^{1}\right)\left(1 h^{1} 2 h^{1}\right)_{T_{0}} \rightarrow\left(2 h^{1}\right)$ which is not shown here] and Fig. 1 we obtain $\Delta_{1 h-2 h}=$ $13.70 \pm 0.02 \mathrm{meV}$.

Similarly, the difference between the first and second electron orbitals can be determined by inspecting Fig. 4(f). One finds that the energy difference between the doublet in the PLE spectrum due to the optical transitions $\left(1 e^{1}\right) \rightarrow\left(1 e^{1} 2 e^{1}\right)_{T_{0}}\left(1 h^{1}\right)$ and $\left(1 e^{1}\right) \rightarrow\left(1 e^{1} 2 e^{1}\right)_{T_{ \pm 1}}\left(1 h^{1}\right)$ and the doublet in PL due to the optical transitions $\left(1 e^{1} 2 e^{1}\right)_{T_{0}}\left(1 h^{1}\right) \rightarrow\left(2 e^{1}\right)$ and $\left(1 e^{1} 2 e^{1}\right)_{T_{ \pm 1}}\left(1 h^{1}\right) \rightarrow\left(2 e^{1}\right)$ is exactly $\Delta_{1 e-2 e}=27.85 \pm$
$0.02 \mathrm{meV}$.

We note that the first excited state of the negative trion is a singlet [see Fig. 4(e)] while the first one of the positive trion is a triplet see Fig. 4(d)]. This difference is expected since the lowest energy excited resonance is obtained by promoting a heavy hole, rather than an electron (Fig. 3). This further supports our spectroscopic identification.

The symmetry-allowed optical transitions between the second electron and hole states described in Fig. 3(c) give rise to very strong resonances in the various PLE spectra (colored red). We note however that the linewidth of these symmetry-allowed transitions differ significantly. While the line-width of resonances to states in which the two electrons form triplets is very narrow, transitions to states in which there is only single electron in the second electron state, or to states in which the two electrons form singlets, are quite broad. We attribute this broadening to optical phonon-induced strong coupling between the first and second electronic orbitals. The LO phonon dispersion broadens these transitions and enhances their optical strength. Since the phonon does not mix states of different electronic spin, triplet electronic states are not coupled to the ground singlet state. We note that 


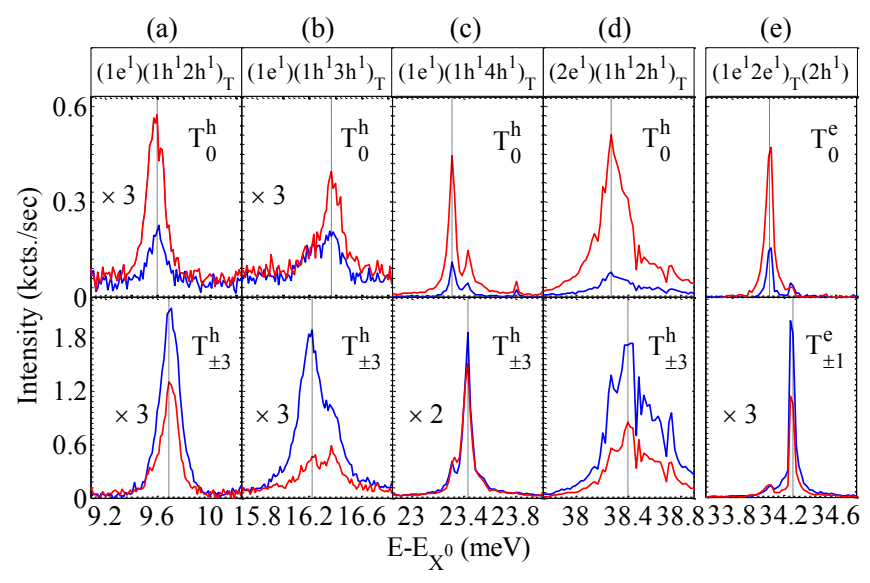

FIG. 5: $\mathrm{HH}$ (blue line) and VV (red line) rectilinearly polarized PLE spectra, where the first (second) letter indicates the polarization of the exciting (emitted) light. The following resonances are presented: (a) $\left(1 e^{1}\right)\left(1 h^{1} 2 h^{1}\right)_{T}$, (b) $\left(1 e^{1}\right)\left(1 h^{1} 3 h^{1}\right)_{T},(\mathrm{c})\left(1 e^{1}\right)\left(1 h^{1} 4 h^{1}\right)_{T}$, (d) $\left(2 e^{1}\right)\left(1 h^{1} 2 h^{1}\right)_{T},(\mathrm{e})$ $\left(1 e^{1} 2 e^{1}\right)_{T}\left(2 h^{1}\right)$.

these resonances are about $29 \mathrm{meV}$ higher than the corresponding resonances in which the electron is in the first level. This energy separation characterizes the energy of LO phonons in compounds of GaAs and InAs! 37 40

The phonon induced strong coupling also enables the symmetry forbidden optical transition that results in photogeneration of a $\left(2 e^{1}\right)\left(1 h^{1}\right)$ electron-hole pair $\underline{36}$ These transitions become strong and broad as marked yellow in Fig. 4(a,e). Their energy difference is about 29 meV higher than the corresponding transition into the first electronic orbital.

In Fig. 5, the rectilinearly polarized PLE spectra of a few of the resonances observed in Fig. 4(d) and (f) are displayed. The resonances appear in pairs since they result from the triplet hole states $T_{0}^{h}$ and $T_{ \pm 3}^{h}$ [Fig. 5(ad)] and from the triplet electron states $T_{0}^{e}$ and $T_{ \pm 1}^{e}$ Fig. 5(e)]. Spectra in which the exciting light is polarized $\mathrm{H}$ (V) and the detection is polarized $\mathrm{H}(\mathrm{V})$ are displayed in blue (red).

We note that, due to the anisotropic electron-hole exchange, the two triplet states are mixed and therefore partially linearly polarized as schematically described in Fig. 2. This partial linear polarization holds also in Fig. 5(d) despite the LO-phonon induced spectral broadening. As mentioned above, phonon mediated transitions preserve the electronic spin and therefore also preserve the optical polarization. Additionally, in all the spectra except the one presented in Fig. 5(b) the higher energy transition among the doublet, is polarized $\mathrm{H}$ and leads into the triplet states in which the majority carriers' spins are aligned $\left(T_{ \pm 3}^{h}\right.$ and $T_{ \pm 1}^{e}$ for holes and electrons, respectively). In Fig. 5(b), however, this energy order is reversed. We attribute this reversal to the difference in the symmetry of the excited hole state, which is $p_{V}$-like, rather than $p_{H}$-like or $d_{H H^{-}}$-like in all the other cases. This symmetry difference results in the sign reversal of $\widetilde{\Delta}$ in Fig. 2.

\section{SUMMARY}

In summary, we present a comprehensive study of the optical transitions of the same single self assembled quantum dot in various charge states. Our study provides a systematic way of understanding the rich photoluminescence and photoluminescence excitation spectra that such quantum dots reveal and in particular, a direct measurement of the confined single carriers' energy level separations. The experimental and theoretical tools that we developed for the spectroscopic characterization of these variably charged quantum dots are essential for achieving coherent control of carrier spins in semiconductor quantum dots and for possible implementation of quantum logic.

\section{Acknowledgments}

The support of the US-Israel Binational Science Foundation (BSF), the Israeli Science Foundation (ISF), the Israeli Ministry of Science and Technology (MOST), Eranet Nano Science Consortium and that of the Technion's RBNI are gratefully acknowledged.
* Electronic address: byael@tx.technion.ac.il

1 T. Takagahara, Phys. Rev. B 62, 16840 (2000).

2 I. A. Akimov, K. V. Kavokin, A. Hundt, F. Henneberger, Phys. Rev. B. 71, 075326 (2005).

3 E. Poem, J. Shemesh, I. Marderfeld, D. Galushko, N. Akopian, D. Gershoni, B. D. Gerardot, A. Badolato, P. M. Petroff, Phys. Rev. B 76, 235304 (2007).

4 M. Ediger, G. Bester, A. Badolato, P. M. Petroff, K. Karrai, A. Zunger, R. J. Warburton, Nature Physics 3, 774 (2007).

5 T. Warming, E. Siebert, A. Schliwa, E. Stock, R. Zimmermann, D. Bimberg, Phys. Rev. B 79, 125316 (2009).
${ }^{6}$ Y. Kodriano, E. Poem, N. H. Lindner, C. Tradonsky, B. D. Gerardot, P. M. Petroff, J. E. Avron, D. Gershoni, Phys. Rev. B 82, 155329 (2010).

7 Y. Benny, Y. Kodriano, E. Poem, S. Khatsevitch, D. Gershoni, P. M. Petroff, Phys. Rev. B 84, 075473 (2011).

8 P. Zanardi, F. Rossi, Phys. Rev. Lett. 81, 4752 (1998).

9 A. Imamoḡlu, D. D. Awschalom, G. Burkard, D. P. DiVincenzo, D. Loss, M. Sherwin, A. Small, Phys. Rev. Lett. 83, 4204 (1999).

10 D. Press, K. De Greve, P. L. McMahon, T. D. Ladd, B. Friess, C. Schneider, M. Kamp, S. H“fling, A. Forchel, Y. Yamamoto, Nature Photonics 4, 367 (2010). 
11 N. Akopian, N. H. Lindner, E. Poem, Y. Berlatzky, J. Avron, D. Gershoni, B. D. Gerardot, P. M. Petroff, Phys. Rev. Lett. 96, 130501 (2006).

12 F. De Martini, G. Di Giuseppe, M. Marrocco, Phys. Rev. Lett. 76, 900 (1996).

13 P. Michler, A. Kiraz, C. Becher, W. V. Schoenfeld, P. M. Petroff, L. Zhang, E. Hu, A Imamoglu, Science 290, 2282 (2000).

14 O. Benson, C. Santori, M. Pelton, Y. Yamamoto, Phys. Rev. Lett. 84, 2513 (2000).

15 D. Fattal, K. Inoue, J. Vučković, C. Santori, G. S. Solomon, Y. Yamamoto, Phys. Rev. Lett. 92, 037903 (2004).

16 O. D. D. Couto Jr., J. Puebla, E. A. Chekhovich, I. J. Luxmoore, C. J. Elliott, N. Babazadeh, M. S. Skolnick, A. I. Tartakovskii, A. B. Krysa, Phys. Rev. B 84, 125301 (2011).

17 R. J. Warburton, C. Schäflein, D. Haft, F. Bickel, A. Lorke, K. Karrai, J. M. Garcia, W. Schoenfeld, P. M. Petroff, Nature 405, 926 (2000).

18 D. Heiss, V. Jovanov, M. Caesar, M. Bichler, G. Abstreiter, J. J. Finley, App. Phys. Lett. 94, 072108 (2009).

19 A. Barenco, M. A. Dupertuis , Phys. Rev. B 52, 2766 (1995).

20 E. Dekel, D. Gershoni, E. Ehrenfreund, J. M. Garcia, P. M. Petroff, Phys. Rev. B 61, 11009 (2000).

21 E. Poem, Y. Kodriano, C. Tradonsky, B. D. Gerardot, P. M. Petroff, and D. Gershoni, Phys. Rev. B 81, 085306 (2010).

22 S. Alon-Braitbart, E. Poem, L. Fradkin, N. Akopian, S. Vilan, E. Lifshitz, E. Ehrenfreund, D. Gershoni, B. D. Gerardot, A. Badolato, P. M. Petroff, Phisica E 32, 127 (2006).

23 E. Siebert, T. Warming, A. Schliwa, E. Stock, M. Winkelnkemper, S. Rodt, D. Bimberg, Phys. Rev. B 79, 205321 (2009).

24 Y. Benny, S. Khatsevich, Y. Kodriano, E. Poem, R. Presman, D. Galushko, P. M. Petroff, D. Gershoni, Phys. Rev. Lett. 106, 040504 (2011).

${ }^{25}$ H. Benisty, H. De Neve, C. Weisbuch, IEEE J. QE 34, 1612 (1998).

${ }^{26}$ G. Ramon, U. Mizrahi, N. Akopian, S. Braitbart, D. Gershoni, T. L. Reinecke, B. Gerardot, P. M. Petroff, Phys.
Rev. B 73, 205330 (2006).

27 J. J. Finley, A. D. Ashmore, A. Lemaître, D. J. Mowbray, M. S. Skolnick, I. E. Itskevich, P. A. Maksym, M. Hopkinson, T. F. Krauss, Phys. Rev. B 63, 073307 (2001).

28 M. E. Ware, E. A. Stinaff, D. Gammon, M. F. Doty, A. S. Bracker, D. Gershoni, V. L. Korenev, S. C. Badescu, Y. Lyanda-Geller, T. L. Reinecke, Phys. Rev. Lett. 95, 177403 (2005).

29 A. Hartmann, Y. Ducommun, E. Kapon, U. Hohenester, E. Molinari, Phys. Rev. Lett. 84, 5648 (2000).

30 D. V. Regelman, D. Gershoni, E. Ehrenfreund, W. V. Schoenfeld, P. M. Petroff, Phys. Stat. Sol. 190, 491 (2002).

31 E. Poem, Y. Kodriano, C. Tradonsky, N. H. Lindner, B. D. Gerardot, P. M. Petroff, D. Gershoni, Nat. Phys. 6, 993 (2010).

32 V. K. Kalevich, I. A. Merkulov, A. Yu. Shiryaev, K. V. Kavokin, M. Ikezawa, T. Okuno, P. N. Brunkov, A. E. Zhukov, V. M. Ustinov, Y. Masumoto, Phys. Rev. B 72, 045325 (2005).

33 K. V. Kavokin, Phys. Stat. Sol. 195, 06157 (2003).

34 E. L. Ivchenko, G. E. Pikus, Superlattices and other Heterostructures (Springer-Verlag, Berlin, 1997).

35 D. Gammon, E. S. Snow, B. V. Shanabrook, D. S. Katzer, D. Park, Phys. Rev. Lett. 76, 3005 (1996).

36 S. Hameau, Y. Guldner, O. Verzelen, R. Ferreira, G. Bastard, J. Zeman, A. Lemaître, J.M. Gérard, Phys. Rev. Lett. 83, 4152 (1999).

37 D. Sarkar, H. P. van der Meulen, J. M. Calleja, J. M. Becker, R. J. Haug, K. Pierz, Phys. Rev. B 71, 081302 (2005).

38 D. Sarkar, H. P. van der Meulen, J. M. Calleja, J. M. Meyer, R. J. Haug, K. Pierz, Appl. Phys. Lett. 92, 181909 (2008).

39 A. Lemaître, A. D. Ashmore, J. J. Finley, D. J. Mowbray, M. S. Skolnick, M. Hopkinson, T. F. Krauss, Phys. Rev. B 63, 161309 (2001).

40 F. Findeis, A. Zrenner, G. Böhm, G. Abstreiter, Phys. Rev. B 61, R10579 (2000). 